\title{
Finite Element Analysis of CNC Lathe Bed
}

\author{
Zhang Weiwei, Kong Jiali \\ School of Mechanical Engineering, Shandong Huayu University of Technology, Dezhou 253034, China \\ 79483895@qq.com
}

Keywords: lathe bed, finite element, model analysis.

\begin{abstract}
For lathe designing, it needs strict calculation and analysis of the major components. The dynamic stiffness of the structure is studied in order to resist deformation under dynamic load, the structure of the dynamic stiffness is improved to enhance the anti-vibration capability of the body. Then the dynamic stiffness and the natural frequency of the structure are measured. Finite element analysis, that affect the working accuracy of the whole machine, is carried out. Simulation results show that the maximum amplitude of vibration attenuation is smaller, faster, and the dynamic stiffness is greater, which can enough support parts of the whole machine tool.
\end{abstract}

\section{Introduction}

With the development of the industry of metal cutting, machine tools have become increasingly demanding[1]. Mechanical work such as machine tools and general machinery and other complex mechanical systems, often affected by the vibration, noise and other problems, which improve the dynamic characteristics of the structure, it is the main task of the structural optimization[2][3]. The modular design of mechanical structure based on finite element analysis in the paper. Through the optimization of the structure, not only the static performance of parts is improved, but also it make the anti-vibration characteristics of the structure is improved, and material is saved, it obtain the optimal structure size finally. Finally, through computer simulations, they shows that the maximum amplitude of vibration attenuation is smaller and the dynamic stiffness is better.

\section{Establishment of Finite Element Model of Lathe Bed}

\subsection{Structural Modeling of Bed}

As we all know, choosing the best modeling method is the key to establish accurate model. In this paper structure model is established by 3D modeling software Solidworks, and then the structure model established in Solidworks by the interface function into ANSYS so as to establish the finite element model of lathe bed. The structure of the bed parts is shown in Figure 1.The finite element model of the bed is missing in the round chamfer, CAD model, all the characteristics of small holes, in addition, it can not account for the whole static, dynamic characteristics of influence of figure CAD model of the simplified parts[4]. The lathe bed structure is shown in Figure 2.

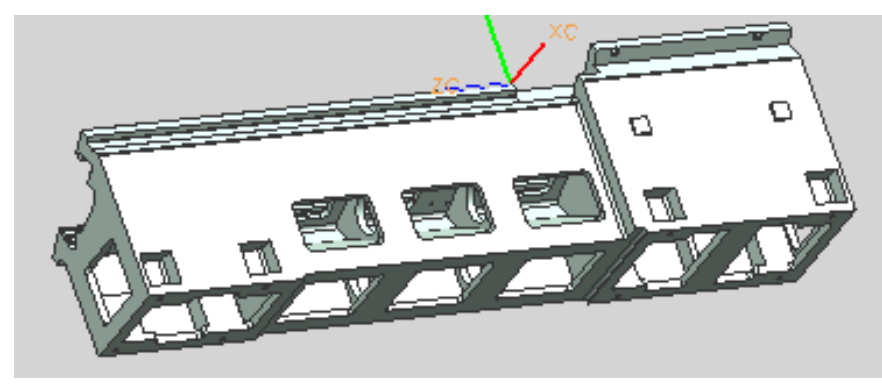

Figure 1 Structure of bed 


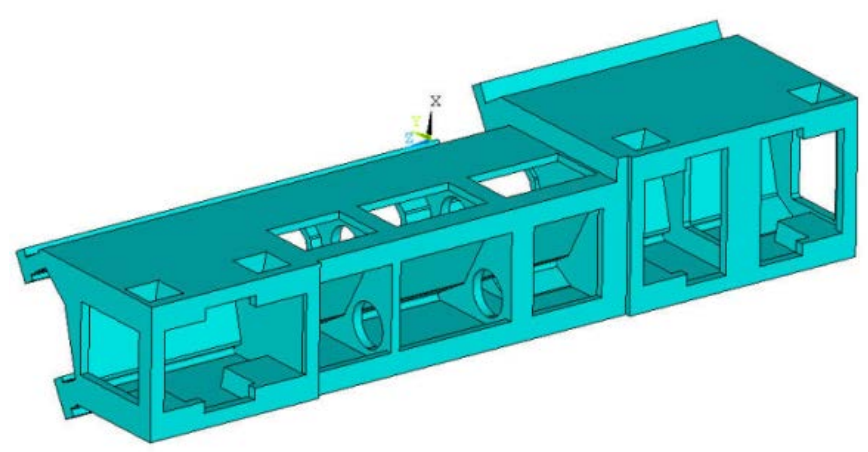

Figure 2 Sketch of bed structure

\subsection{Establishment of Finite Element Model of Lathe Bed}

According to the characteristics of machine tool bed system's modeling and structural mechanics, machine tool bed with SOLID285 unit are obtained. SOLID285 unit are used for irregular mesh model, and the displacement function of the unit can been seen from formula(1),formula(2) and formula(3). 4 node tetrahedral elements, shown in Figure 3. Each node has three degrees of freedom: $\mathrm{X}, \mathrm{Y}, \mathrm{Z}$ direction[4]. The material for the lathe bed is HT250, and its modulus, density and Poisson's ratio are shown in Table 1 . When the bed is discretized, the length of the unit is taken $15 \mathrm{~mm}$. The lathe bed is directly crouching on the legs of the bed and introduced into the CAD model of the spindle in the ANSYS. The 3 degree of freedom constraint is applied to the bottom surface. The finite element model established here is shown in Figure 4.

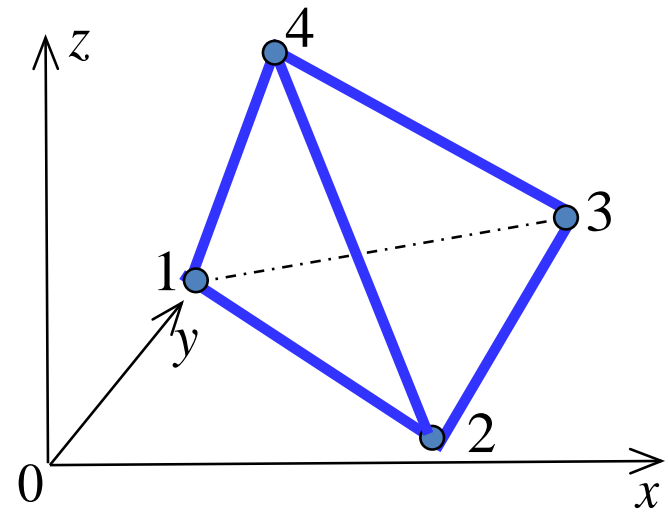

Figure 34 node tetrahedron element

$$
\begin{aligned}
& \mu=\partial_{1}+\partial_{2} x+\partial_{3} y+\partial_{4} z \\
& v=\partial_{5}+\partial_{6} x+\partial_{6} y+\partial_{7} z \\
& w=\partial_{9}+\partial_{10} x+\partial_{11} y+\partial_{12} z
\end{aligned}
$$

Table 1 Material related parameters[5]

\begin{tabular}{|c|c|c|}
\hline Modulus of elasticity $E(P a)$ & Density $\rho\left(\mathrm{kg} / \mathrm{m}^{3}\right)$ & Poisson ratio $\mu$ \\
\hline 140 & 7340 & 0.25 \\
\hline
\end{tabular}




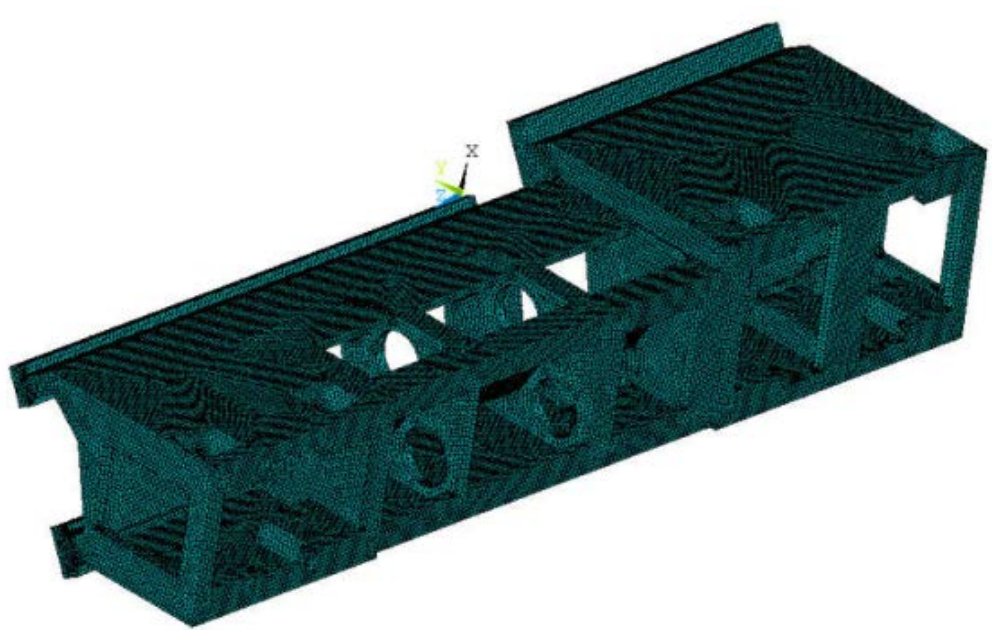

Figure 4 Finite element model of the bed

\section{Natural Frequencies and Mode Analysis}

When the bed is discretized, the length of the unit is $15 \mathrm{~mm}$, the total number of nodes is 58298 , the total number of units is 218298., and the natural frequency of the first six steps are shown in Table 2. In this paper, it use the recursive formula to produce an orthogonal vector matrix, which is one of the most effective methods for solving large matrix eigenvalue problems, that is, the Block Lanczos method. The linear superposition of various modes can express the vibration of the structure, the dynamic characteristics of low order modes of structure play a decisive role, because of the low order vibration mode than the effect of higher modes of vibration on the structure, the analysis of the vibration characteristics of the structure, usually from 5 to 10 . The order can be the modal analysis of machine tool bed when the first six order modal shapes, as shown in Figure 5. It can be seen from Figure 5 that these six order vibration patterns that the bending deformation is obvious due to the influence of the main cutting force on the part and the tail of the tool post.

Table 2 Natural vibration frequency of the bed

\begin{tabular}{|c|c|}
\hline Order & Natural frequency/ Hz \\
\hline 1 & 304.10 \\
\hline 2 & 339.13 \\
\hline 3 & 413.98 \\
\hline 4 & 509.29 \\
\hline 5 & 560.29 \\
\hline 6 & 612.17 \\
\hline
\end{tabular}

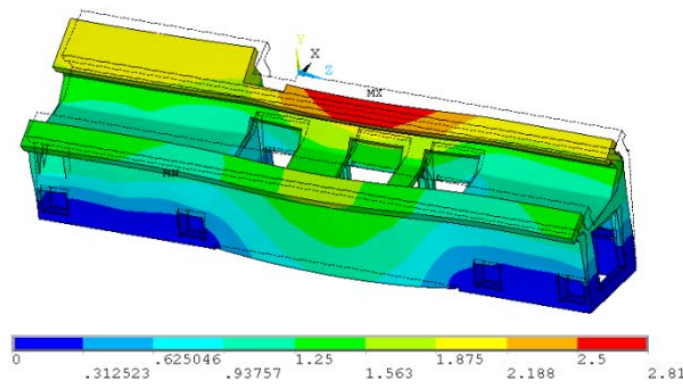

a)First order vibration pattern

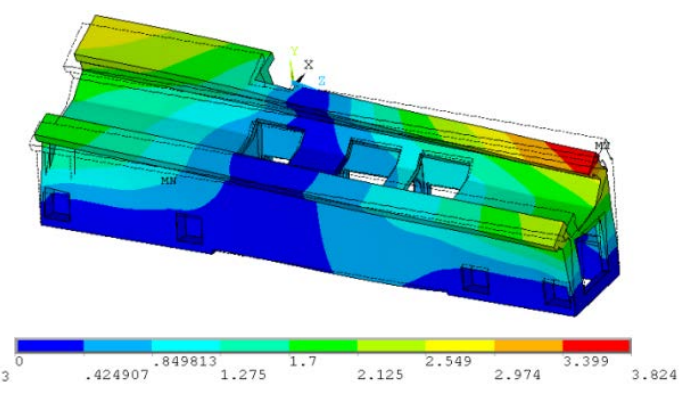

b)Two order vibration pattern 


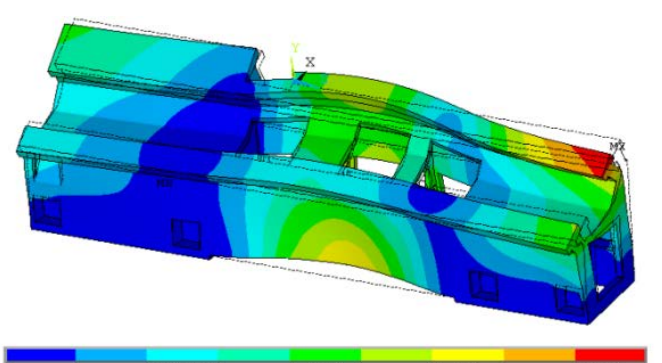

Three order vibration pattern

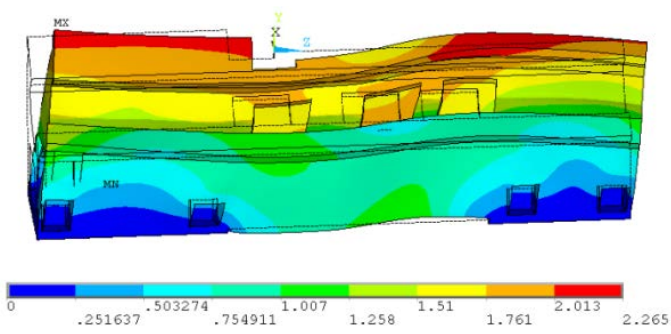

e)Five order vibration pattern

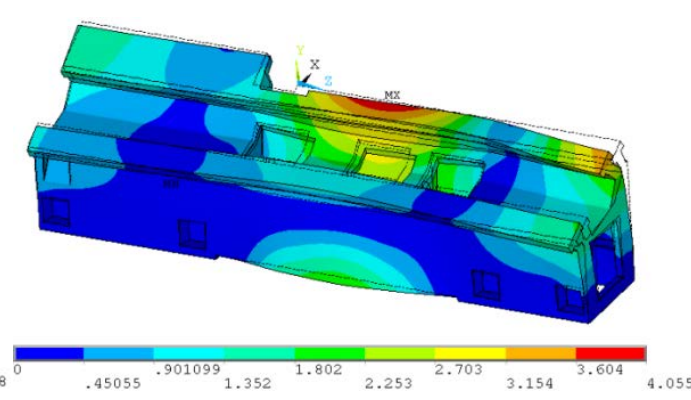

d) Four order vibration pattern

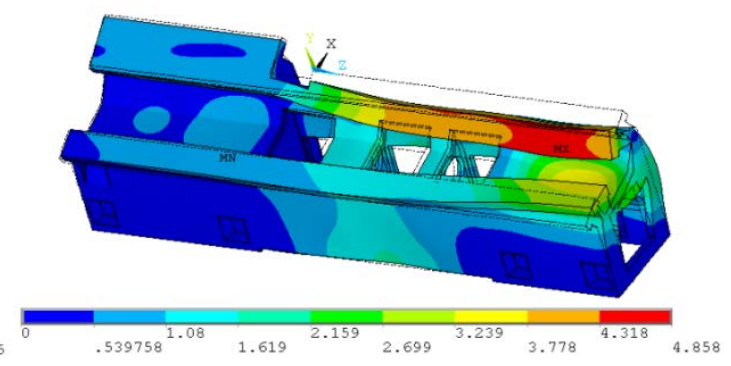

f) Six order vibration pattern

Figure 5 Vibration pattern of the first 6 orders

\section{Conclusions}

It can be seen from the above analysis, the original model can meet the requirements of static stiffness required for production. It has good dynamic characteristics in low order frequency, the vibration frequency is $304.10 \mathrm{~Hz}$, in the machine tool working speed. The overall vibration of the first four order in the bed is small and the maximum amplitude is consistent, therefore, the structure accords with the the dynamic stiffness of the design requirements is perfect.

\section{References}

[1] Wen G. Z., Wang G., Wang J., etc. (2013) Dynamic analysis of rotating shaft of test machine based on finite element method. Mechanical design and manufacturing.

[2] Kong J. L. (2017) Finite element analysis of CNC lathe bed. Science and Technology Economy.

[3] Yang J. H., Huang Y. D., Fei R.Y. (1993)Vibration modal analysis of spindle assembly. Journal of Beijing University of Technology,19(2): 89-95.

[4] Kong J. L. (2017) The design of simple slant bed horizontal CNC lathe. Tianjin University of Technology and Education, 42-49.

[5] Liu H. W. (2010) Mechanics of materials[M].Beijing: Higher Education Press,241-246. 